\title{
Individual differences in collinearity judgment as a function of angular position
}

\author{
ERNEST GREENE \\ University of Southern California, Los Angeles, Califormia, \\ and Neuropsychology Foundation, Los Angeles, California \\ WILLLAM FRAWLEY \\ University of Texas, Southwest Medical Center, Dallas, Texas \\ and \\ RANDALL SWIMM \\ Neuropsychology Foundation, Los Angeles, Califormia
}

\begin{abstract}
Previous research indicates that perceived orientation and/or alignment of segments and points can vary as a function of the angular position of the stimulus elements. Several studies show that the variability of the responses is least and accuracy of judgment is greatest where segments and dots are aligned with a cardinal axis. Additionally, some report assimilation of judgments toward the nearest cardinal axis - that is, the segments (or dots) are seen as being closer to the horizontal or vertical than is true. The present research confirms that judgments of collinearity are least variable and most accurate when the segment being judged is aligned with a cardinal axis. However, we do not find any consistent tendency for cardinal axis assimilation. Plotting the collinearity error (delta) as a function of angular position (phi), we find a distinctive profile of oscillation for each subject. Furthermore, subjects who were evaluated in two sessions showed very similar profiles of delta oscillation from Day 1 to Day 2. Harmonic analysis indicated a wide-ranging pattern of significant components. The components at the 4th harmonic and below were more likely to be significant, but each subject showed differential loadings in terms of which of the components were significant, as well as in the sign and amplitude of significant components. These results may reflect idiosyncratic fixation tendencies, or individual differences in the design of neural mechanisms that encode the angular positions of stimuli.
\end{abstract}

Numerous studies during the past century have documented that perception of line segments and gratings depends on their alignment relative to the cardinal axes ${ }^{1}$ of the observer (i.e., the horizontal and vertical axes of the visual field). Jastrow (1893) tested the accuracy of orientation judgment, asking subjects to view a segment and then immediately (from memory) set another segment to lie at the same orientation. He found that there was less variability in the judgments of horizontal or vertical segments, and the judgments were more accurate.

Superior orientation discrimination for single segments has also been reported by Vernon (1934), Andrews (1967), and Orban, Vandenbussche, and Vogels (1984). Craig and Lichtenstein (1953) reported that oblique segments that were viewed with steady fixation disappeared more readily and for longer durations than did horizontal or vertical segments. Leibowitz, Meyers, and Grant (1955) found that single dots that were flashed to an eccentric position

This research was sponsored, in part, by the Neuropsychology Foundation, Los Angeles. The authors thank Brian Nelson for testing some subjects. Correspondence should be addressed to Ernest Greene, Department of Psychology, University of Southern California, Los Angeles, California 90089-1061 (e-mail: egreene@bcf.usc.edu). (i.e., relative to fixation) were more accurately localized if they were positioned on or near a cardinal axis. Emsley (1925), Leibowitz (1953), and Regan and Price (1986) found that grating acuity was better for horizontal and vertical orientations than when the gratings were at a tilt.

The superior accuracy, perceptibility, and discrimination thresholds reported above are accompanied by differentials in variance. For example, Sulzer and Zener (1953) found variability in orientation judgments to be minimal for horizontal segments, near minimal for vertical segments, and maximal for oblique segments. Rochlin (1955) and Onley and Volkmann (1958) report similar effects. All the differentials enumerated above were discussed by Appelle (1972), who identified them collectively as the "oblique effect."

In addition to these oblique effects, there is evidence that judgment of orientation and angular position ${ }^{2}$ may be systematically biased toward or away from the nearest cardinal axis, with the former being described as assimilation and the latter as contrast. ${ }^{3}$ Jastrow (1893) also reported a bias in the phenomenal positioning of horizontal, vertical, and oblique segments (i.e., relative to the eyes) with head upright and using gravity as a reference. Again, the horizontal and vertical settings were more accurate and less variable, but, in addition, there was a tendency for 
oblique segments to be set too close to the horizontal by a few degrees. Although he did not use the term, we would now describe this as assimilation toward the horizontal axis.

Bouma and Andriessen (1968) also evaluated single line segments, asking the subjects to demonstrate their perception of angular position by means of a collinearity judgment - that is, by moving the position of a remote dot until it appeared to be aligned with the segment. The main study tested 3 subjects, who were asked to judge angular position within one quadrant. These investigators found minimal variability in judgments of horizontal and vertical positions and found maximal variability at $45^{\circ}$. They also found a systematic pattern of error across the angular positions that were tested, with the basic effect being an assimilation toward the nearest cardinal axis.

Bouma and Andriessen (1968) provided formal models of these results. Variability was modeled as a cycloid, and misperceived alignment was given as a 4th harmonic sine wave. As a test of the sinusoidal model, Bouma and Andriessen conducted a second experiment, wherein 2 subjects judged eight angles (across four quadrants), testing the angles at which the model predicts maximal error. The results show substantial departure from accurate judgment at each of the eight angles, more or less consistent with the 4 th harmonic bias that was specified by their model.

Smith (1962) tested 10 subjects in a task that required setting a rotary switch to an angle that matched the path of a simulated radar trail (consisting of a string of dots). He also found more accurate judgments at cardinal axes and fair accuracy at the $45^{\circ}$ oblique, but he reported that, for intermediate angles, there was a tendency to judge the angle as lying less close to the cardinal axis. In other words, Smith found contrast as a function of proximity to a cardinal axis, rather than assimilation.

Sittig and De Graaf (1994) evaluated collinearity judgments as well, using a three-dot configuration and requiring adjustment of the center dot. In one experiment, they tested 8 subjects at 32 angular positions across the full $360^{\circ}$ range and plotted the data of the individual subjects. On average, the only consistent effect was a tendency for assimilation toward the horizontal axis, seen only in the upper quadrants.

In addition to these direct psychophysical studies, it should be noted that a number of investigators who work with visual illusions have proposed that illusion strength is a function of horizontal/vertical alignment (e.g., Judd, 1899; Weintraub \& Krantz, 1971). Furthermore, some have argued that a portion of illusion effect is due to assimilation toward a cardinal axis. Wundt (1893) first reported that one sees a Poggendorff-like illusion even when an oblique segment is presented alone, and there are no parallels to produce a bias in judgment. He attributed the effect to an assimilation of the segment being judged toward either the vertical axis or the horizontal axis of the observer. Green and Hoyle (1964), Hotopf, Ollerearnshaw, and Brown (1974), and Weintraub and Virsu (1971) have made similar suggestions.

Thus, some studies indicate that there is assimilation of collinearity judgment toward the nearest cardinal axis, whereas others find assimilation to the horizontal axis only, minimal effects, or even contrast. It does not appear that the direction, or even the existence of, assimilation toward a cardinal axis has been settled. Therefore, we conducted two experiments in which the angular position of a segment was varied, and, for each angle, the subject was asked to make a collinearity judgment (i.e., placing a dot on the sheet so that it appeared to be aligned with the segment). The results of both experiments support the proposition that horizontal and vertical judgments are less variable and more accurate, but there was no indication of cardinal axis assimilation. Rather, the plot of errors showed aperiodic oscillations for each subject, each having a distinctive profile (shape) that was essentially the same from one test day to the next. We describe these oscillations as a manifestation of "intrinsic bias," in that the error is not being produced by interactions among stimulus segments, as is true for most geometric illusions.

\section{METHOD}

\section{Subjects}

Twenty USC undergraduates were paid for their participation in the experiment. Each was tested for acuity using a Snellen Eye Chart, and each was found to have $20 / 20$ vision (some subjects having the benefit of contact-lens correction). The subjects were naive with respect to the phenomenon under investigation. Eight were tested in Experiment 1 and are designated as Subjects 1-8. Twelve were tested in Experiment 2 and are designated as Subjects 9-20.

\section{Stimulus Materials}

The stimulus materials consisted of a set of white $8.5 \times 11$ in. test sheets, each displaying a black stimulus segment, designated as the test segment. The angular position of the segment, designated as phi $(\phi),{ }^{4}$ was varied across the sheets of the set. For all sheets, the test segment was $0.54 \mathrm{~mm}$ thick and $6 \mathrm{~cm}$ long, which correspond to visual angles of $4^{\prime}$ of arc and $7.4^{\circ}$ of arc, respectively, under the viewing conditions described below.

Phi and quadrant were defined using standard mathematical convention, with $0^{\circ}$ being a horizontal ray to the right, and increments of angle being counterclockwise. Thus, the first quadrant of the sheet is the upper right, the second is the upper left, the third is the lower left, and the fourth is the lower right, as illustrated in Figure 1.

We did not want to have all segments pivoting about the center of the sheet, since this might provide a common anchor for perceptual judgment. In other words, rather than directly evaluate collinearity in relation to the segment, the subject might simply position the dot at distances from the edge of the sheet that were the same as the distances to the eccentric end of the segment being judged. To avoid this possibility, an eccentric pivot point was used for each quadrant, that point being positioned at $6 \mathrm{~cm}\left(7.4^{\circ}\right.$ of arc) from the center of the sheet and at $45^{\circ}$ angle from the cardinal axes.

For Experiment 1, phi was varied across a $360^{\circ}$ range in $7.5^{\circ}$ increments, presenting each angular position twice within a given quadrant. To provide a complete sampling within each quadrant, the cardinal axes were tested twice. Thus, there were 13 angular positions, 4 quadrants, and 2 replications, for a total of 104 sheets in this test set. 


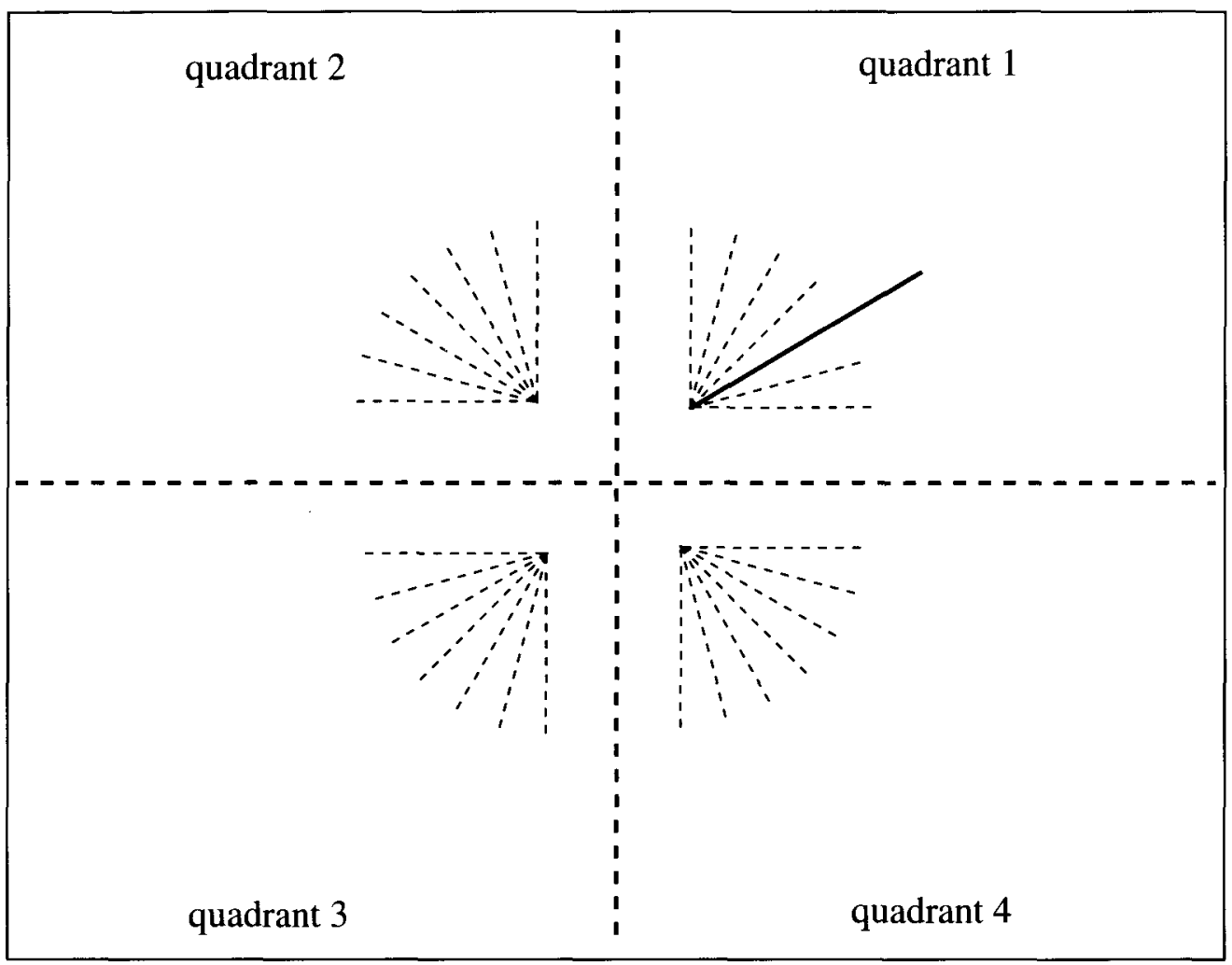

Figure 1. Presentation of the stimulus segment at various angular positions is illustrated, and the quadrants of the test sheet are identified. Within each quadrant, the stimulus segment (shown as a solid black line) was placed at one of the angular positions (illustrated as an array of dashed lines, all increments of Experiment 1 not being shown). Note that the pivot for the rotation within a given quadrant is eccentric to the center of the sheet. None of the dashed lines or quadrant labels were displayed on the stimulus sheets.

For Experiment 2, we wanted to provide a better index of variability at each angular position, so six samples were taken at $15^{\circ}$ increments. Cardinal axes were not redundantly sampled, so the complete test set consisted of 24 angular positions and 6 replications, for a total of 144 sheets.

For each experiment, the sheets of a master test set were printed with a laser printer, having a resolution of $300 \mathrm{dpi}$. The test set that was provided to each subject was a xerographic photocopy of the master set. Each copy was used just once, so the sheets did not contain erasures or any other remnants of prior use.

\section{Apparatus and Test Procedure}

The subjects judged the stimulus sheets with the aid of a viewing stand. The viewing surface of the stand positioned the sheet at a $15^{\circ}$ elevation from the plane of the table and included edge guides to ensure proper alignment of the sheet. The stand also included a mask that positioned the eyes at $46 \mathrm{~cm}$ from the sheet and that adjusted the line of sight to be perpendicular to and centered on the sheet. The horizontal alignment of the two eye holes discouraged twisting of the head and thus helped to preserve the intended angular positions at which the stimulus segments were displayed.

The subjects were tested individually, each being given a test set that had been randomized by shuffling. Each subject was told that this was a test of ability to judge alignment of segments and that the task was to select and mark a point that was "in line" with the test segment. By gesture, a zone approximately $20^{\circ}$ of arc from the tip of the test segment, in an adjacent quadrant, was designated as a suitable target. We describe the subject's mark as the response point.
The subject was told that if the initial mark did not appear to be aligned with the test segment, it could be erased so that the segment could be judged again. Few availed themselves of this option. Once the task requirements had been explained, the subject was allowed to proceed through the test set, successively placing each sheet on the viewing stand, evaluating the angular position of the test segment, and marking a response point. Most finished the task in $45 \mathrm{~min}$ or less.

The data for Experiment 1 were collected in a single test session. For Experiment 2, there were two sessions, each requiring the subject to judge a complete set of test sheets. The second session was scheduled at least 1 day, and no more than 3 days, following the first. Conditions in the second session were identical to the first, except for a different random ordering of the sheets in the test set.

\section{RESULTS}

\section{Operational Definition of Error}

Collinearity error was defined as angular departure of the response point from true collinearity with the segment, measuring the angle from the tip of the stimulus segment that was closest to the response point, as illustrated in Figure 2. Previous studies of angular induction by this laboratory (e.g., Greene, 1993) have found that this measure provides a consistent index of collinearity bias irrespective of the distance at which the subject responds. 


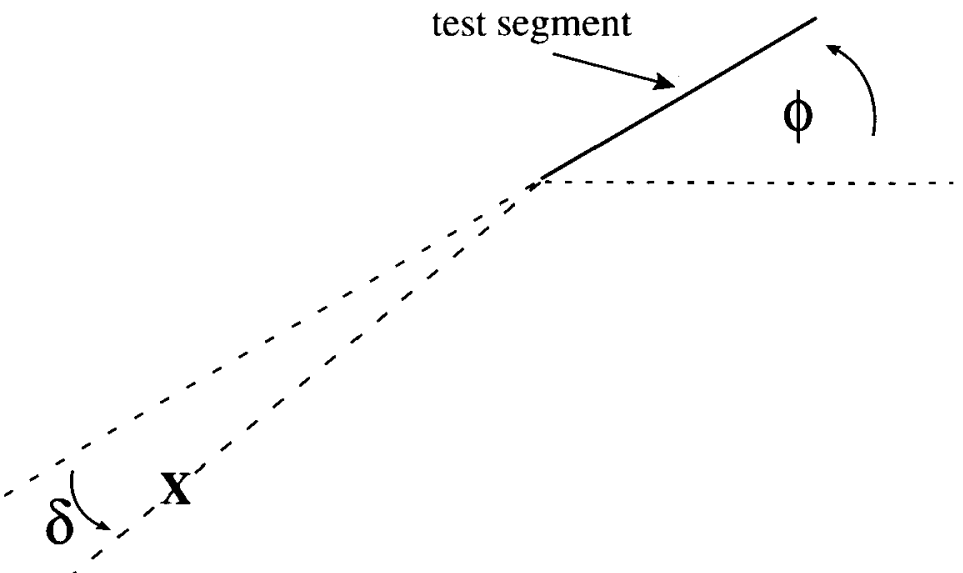

\begin{abstract}
Figure 2. The angular position ( $\phi$ ) was specified as a counterclockwise rotation from a horizontal ray to the right, with the ray beginning at the end of the segment that was closest to the center of the sheet. The subjects were instructed to select a location that appeared to be collinear to the segment, in the open space that was in an adjacent quadrant. The $X$ illustrates a point that might be selected by a subject. Collinearity error $(\delta)$ was measured as an angular departure from true collinearity. For $\phi$ and $\delta$, counterclockwise rotations were designated as positive.
\end{abstract}

In the prior research from this laboratory, the collinearity error was produced by one or more "induction segments," which generated a judgment bias that was designated as psi $(\psi)$. For the present work, there are no additional stimulus elements that might be the source of induction. As a means of distinguishing among these effects, we describe collinearity errors to an isolated test segment as intrinsic bias, and identify it as delta $(\delta)$.

Delta errors were measured to the nearest half degree with the aid of a protractor that had been printed on a transparent sheet, counterclockwise departures from true collinearity being defined as positive.

\section{Not Combining Across Quadrants}

At the outset, it had been our intention to determine whether there were any differences across quadrants and, if not, to get a more reliable summary of effect by combining these data. In Experiment 1, therefore, in each quadrant we sampled the full range of angular positions, including the horizontal and vertical positions at each end of the range. This redundant sampling of cardinal axes was done in order to provide a full complement of angular positions for evaluation of quadrant effect. However, very early in the examination of raw data, it was possible to see a complex pattern of error that was not the same in each quadrant. It became clear, as well, that the very process of combining across quadrants makes assumptions about the sources of bias that may obscure significant effects.

Being more specific, folding of the data is only suitable if a source of judgment bias produces an effect that has a mirror image in adjacent quadrants. Combining the data by rotation does not make this assumption, but it can obscure sources of bias that are lower than the 4th harmonic (one oscillation per quadrant). An omnibus test for whether there are "quadrant differences" would avoid a Type I error (claiming a difference when there was none), but one might be risking a Type II error (falsely concluding that there are no differences).

To avoid these issues, we decided to keep angular positions identified and intact throughout the $360^{\circ}$ range. Thus, the segment that lies at $90^{\circ}$ in Quadrant 1 is considered to be equivalent to the segment that lies at $90^{\circ}$ in Quadrant 2, and likewise for the junction of other quadrants. For Experiment 1, this meant that there was oversampling of cardinal axes relative to other angular positions (i.e., four measures rather than two). For Experiment 2, the cardinal axes within each quadrant were undersampled, so each angular position had an equal number of replications.

\section{Variability of Responses}

The first goal was to evaluate the variability of judgments as a function of angular position. For Experiment 1 , we had only two samples (i.e., replications) for each of these angular positions, which would yield a highly variable estimate of the standard deviation. Therefore, in Experiment 1 , we used a "windowing" technique that added the data on each side of a given phi, thus providing additional values for estimating the standard deviation at that position. That is, for the $0^{\circ}$ estimate, we used observations at $352.5^{\circ}, 0^{\circ}$, and $7.5^{\circ}$; for the $7.5^{\circ}$ estimate, we combined observations at $0^{\circ}, 7.5^{\circ}$, and $15^{\circ}$; and so forth. For Experiment 2, there was a sufficient number of observations at each angular position, so the standard deviation could be calculated directly for each phi. 

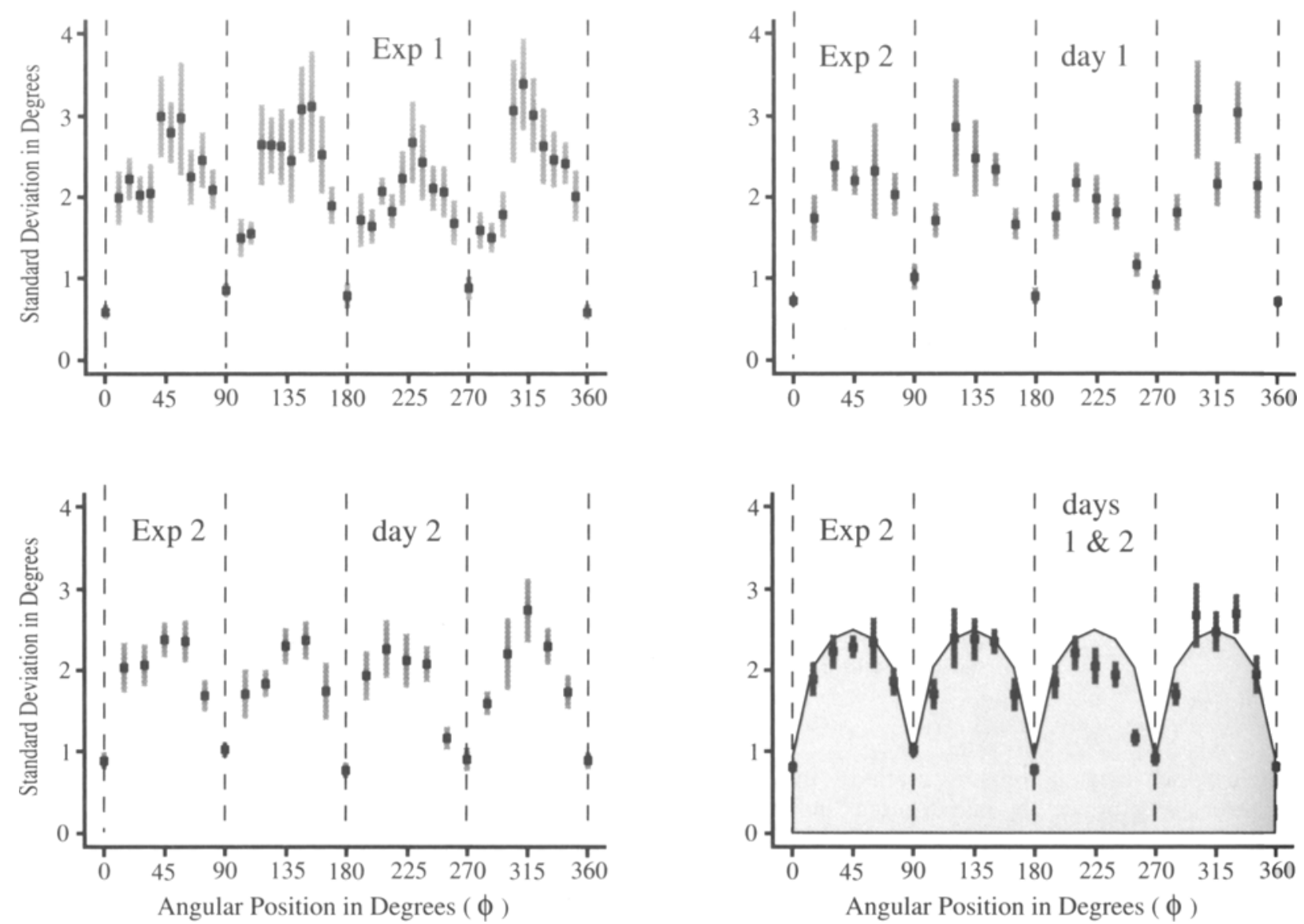

Figure 3. The upper left panel displays the mean and standard error of the standard deviations that were observed at each angular position, taken across the subjects of Experiment 1. The upper right and lower left panels show these values for the two sessions of Experiment 2. In both experiments, it is clear that variability of response is least where the angular position being judged is aligned with a cardinal axis. The lower right panel shows the mean and standard deviation with both sessions combined, superimposed on a model for the effects. The formula for the model is $Y=0.9+1.6[|\sin (2 \phi)|] 1 / 2$.

The standard deviation for each angular position was averaged across subjects, with the means for Experiment 1 being shown in the upper left panel of Figure 3 and the means for the two sessions of Experiment 2 being shown in the upper right and lower left panels. The standard error for each mean is provided by a gray error bar, which designates the $95 \%$ confidence interval. For both experiments, one can see that the mean standard deviation varied as a function of phi. The profile is suggestive of a cycloid, with variability being lowest at cardinal axes. To provide a formal model of this tendency, we combined the averages from both sessions of Experiment 2 and superimposed these values on a cycloid model, as shown in the lower right panel of Figure 3. The parameters for the cycloid were selected on the basis of a subjective judgment of good fit.

\section{Variability: A Comparison Plot of Cardinal Versus Oblique Positions}

The data plots of Figure 3 show smaller standard deviations at angular positions that are aligned with a cardi- nal axis. To better illustrate this differential, we combined the mean values across cardinal versus oblique angular positions and plotted these values in Figure 4. To be specific, the cardinal positions are composed of $0^{\circ}, 90^{\circ}, 180^{\circ}$, and $270^{\circ}$, and the oblique positions are composed of $45^{\circ}$, $135^{\circ}, 225^{\circ}$, and $315^{\circ}$ (with allowance for the windowing method used in Experiment 1, as described above).

Thus, for Experiment 1, we computed the mean standard deviation for cardinal positions and the mean standard deviation for oblique positions, deriving these two measures for each of the 8 subjects. We also derived these measures for the 12 subjects of Experiment 2, calculated separately for each of the two sessions in which they were tested.

The two means occupy a single point in a plot, wherein the oblique-position values are given on the ordinate and the cardinal-position values are given on the abscissa. Thus, each plot point in Figure 4 represents the data of a single subject for one test session. If the measures of variability were the same for cardinal and oblique positions, the collection of plot points would tend to fall along the 


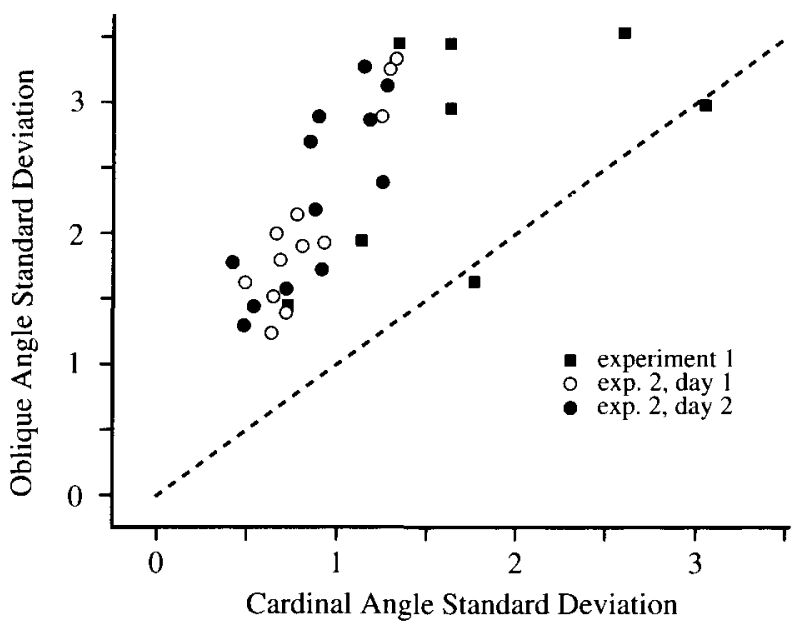

Figure 4. For each subject, one can average across the cardinal angular positions and across the oblique angular positions to derive a mean measure of variability for each set. The plot of one against the other shows that there was consistently higher variability for the oblique angular positions.

diagonal. It is clear that virtually all of the points collect in the upper left corner of the figure, which demonstrates that oblique variability tends to be far higher than does cardinal variability and that this is a consistent result across the pool of subjects.

If the relative size of the two variance estimates were random, one would expect half of the points in Figure 4 to fall above and half to fall below the diagonal. We calculated the odds of observing two or fewer being below the diagonal and found this condition to have a probability of $3.5 \times 10^{-5}$. In other words, we can describe the difference in variability for oblique and cardinal judgments as being significant at $p<.0001$.

\section{Accuracy of Responses: A Comparison Plot of Cardinal Versus Oblique Positions}

As indicated in the introduction, there is also reason to believe that judgments are more accurate when the test segment is aligned with a cardinal axis. We take the absolute magnitude of delta error as indicating the inverse of judgment accuracy, ${ }^{5}$ and we examine this issue by cross-plotting the data for the cardinal versus oblique positions, as described above.

Thus, for each subject, we calculated mean accuracy for cardinal positions and for oblique positions, reflecting these values in the plot points of Figure 5 . As before, we show two points for each subject in Experiment 2, one for the first session and one for the second session. It is clear that the points are clustered in the upper left corner of the figure, indicating that oblique accuracy was far lower than was cardinal accuracy, in that the former had relatively higher levels of judgment error. The odds of this degree of plot asymmetry (i.e., observing all of the points lying above the diagonal) is again $p<.0001$.

We conclude that judgments are more accurate when the test segment is aligned with a cardinal position than when it lies at an oblique position. The greater accuracy can also be observed in the plots of data and the models of effect that are presented below.

\section{Modeling Sources of Intrinsic Bias}

The initial plan was to model intrinsic bias by regressing on the means for the group, focusing especially on the Bouma and Andriessen (1968) hypothesis of cardinal axis assimilation. To be specific, these investigators proposed that the intrinsic bias could be modeled as a 4th harmonic sine function. The data from Experiment 1 were examined with this hypothesis in mind, but there was no clear pattern of effect. At first, we thought that this was due to high error variance, which might be remedied by a large number of replications at each angular position. Only after the completion of Experiment 2 did it become clear that each subject was showing a distinctive profile of errors and that subject-by-subject harmonic analysis would be the most effective way to identify these effects.

Figures 6-8 illustrate how this analysis was applied, using 2 subjects from Experiment 1 and 2 subjects from Experiment 2 (Day 1 only) as examples. The raw data for these subjects have been plotted in Figure 6. In each plot, one can see systematic oscillation of the data points away from the zero line, which specifies accurate judgments. However, for each subject, these delta errors vary idiosyncratically in amplitude and direction as a function of angular position. For Subject 3, the error tends to be counterclockwise in the second quadrant, clockwise in the third, and then swings in both directions in the fourth quadrant. For Subject 4, there are strong counterclockwise departures in the third quadrant, and clockwise departures in the second and fourth quadrants. Subject 9 shows a clear sine oscillation through all four quadrants, whereas Subject 20 has counterclockwise oscillations in Quadrants 1,3, and 4. The profile of these oscillations is

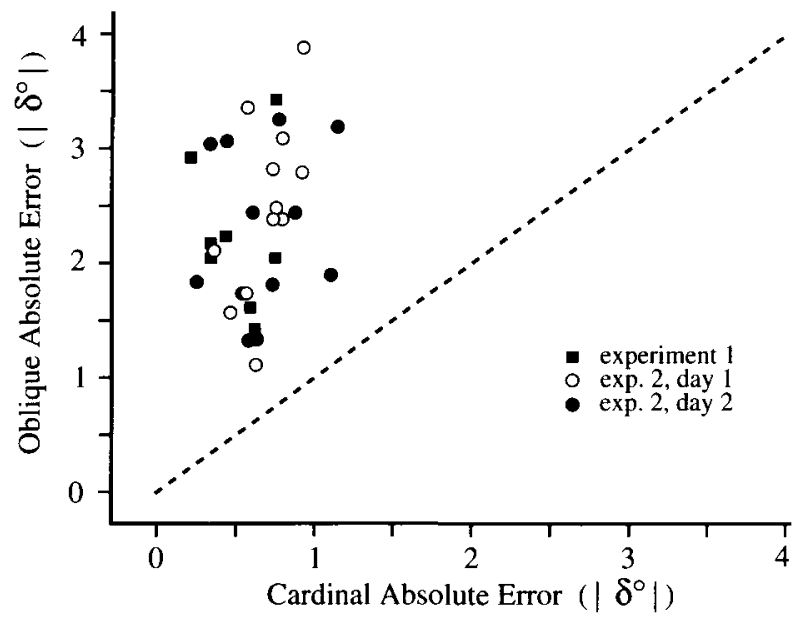

Figure 5. For each subject, one can average across the cardinal angular positions and across the oblique angular positions to derive a mean measure of absolute error. The plot of one against the other shows that judgment errors were consistently higher at oblique angular positions. 

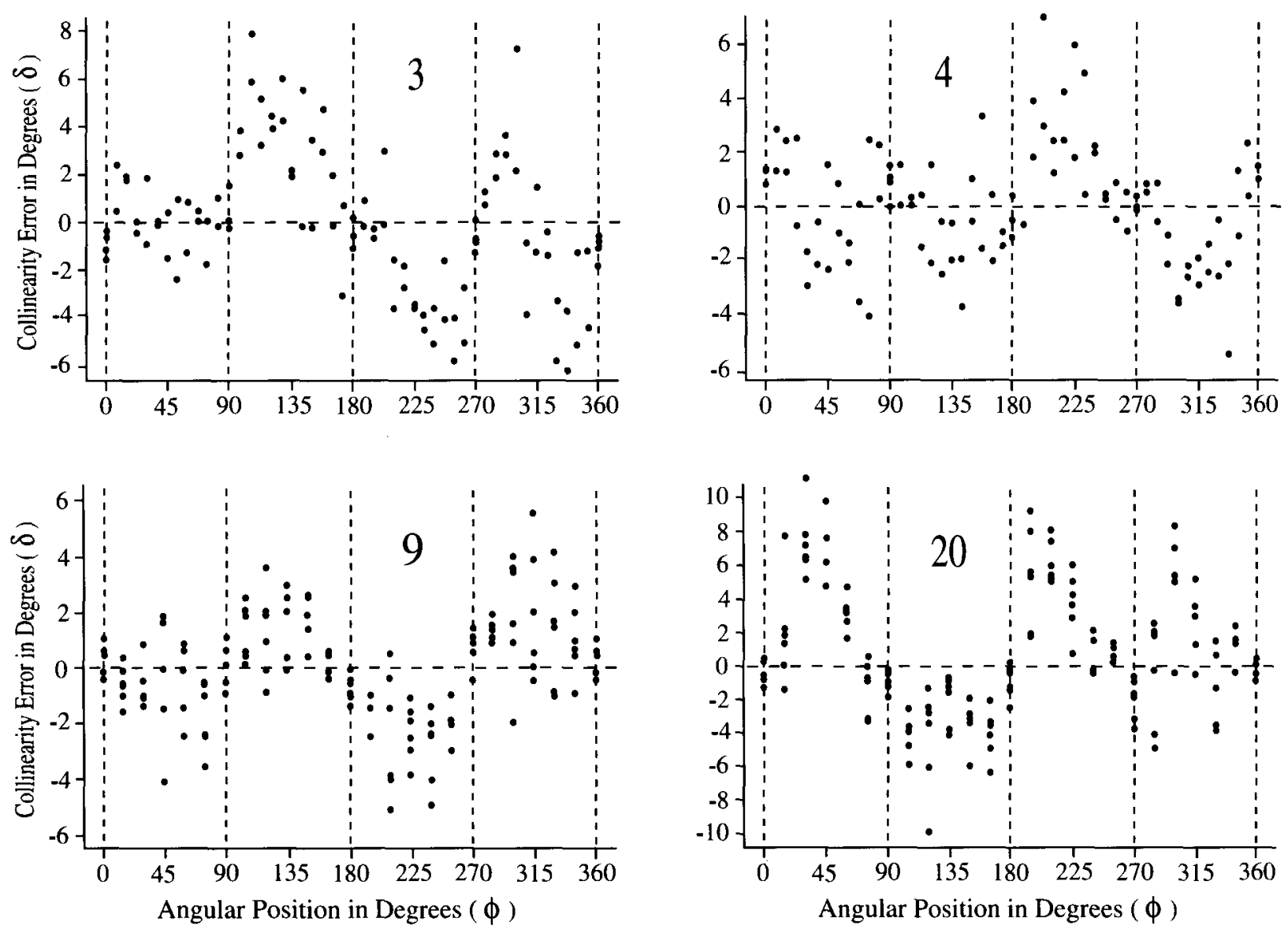

Figure 6. Data plots for 2 subjects of Experiment 1 and for 2 subjects of Experiment 2 (Day 1) are shown in the upper and lower panels, respectively. Systematic departures from accurate judgment can be seen in these data and in the data plots of all other subjects. We refer to these departures as delta oscillations.

especially conspicuous for these 4 subjects, but they are not far from representative of the effects that can be seen across the entire pool of subjects.

For each subject, we performed a harmonic analysis (a Fourier series), weighting observations as an inverse proportion of their estimated variance and extracting the constant and coefficients up to the 12th harmonic. Each bar graph in Figure 7 displays the absolute magnitude of each harmonic component. ${ }^{6}$ For Subject 3 , the sine components of 3 of the first 4 harmonics were all above 1.5 in amplitude; however, for Subject 9, only the sine of the 2nd harmonic reached this level. Subjects 4 and 20 each had a complex profile of significant sine and cosine components, with the largest effects being provided by the first 4 harmonics. Each of these subjects had a distinctive pattern of coefficient amplitudes, and unique profiles could be seen in the plots of other subjects as well. The individual differences would be even more distinctive if the coefficients were plotted as signed values.

On examining the bar graphs of Figure 7, some may find it difficult to understand how the sine and cosine components of a given harmonic could both be significant. This is because the two measures are not opposite but are complementary. Using the 1 st harmonic as an example, each component reflects the degree to which a peak in error tendency falls near the vertical axis or the horizontal axis. The sine and cosine components combine to produce a single sinusoid whose peak is shifted to an angular position that is intermediate between the cardinal axes, with this displacement being described as a phase shift. The degree of shift is determined by the relative amplitudes of the sine and cosine components, so both can be significant if the resultant sinusoid is fairly large and especially if a substantial phase shift is required.

For each subject, the higher amplitude coefficients were then selected for inclusion in a model, meaning that an inverse Fourier transform was calculated using these coefficients. These coefficients were selected using Mallows's Cp statistic (see Hocking, 1976). We included those coefficients that minimized Mallows's Cp statistic. This procedure intuitively can be viewed as a cost function, wherein a penalty is paid (1) if these key coefficients are not included in the model and/or (2) if one includes unimportant coefficients in the model. We compared our coefficient selections with those chosen using other techniques (e.g., a stepwise procedure) and found them to be 

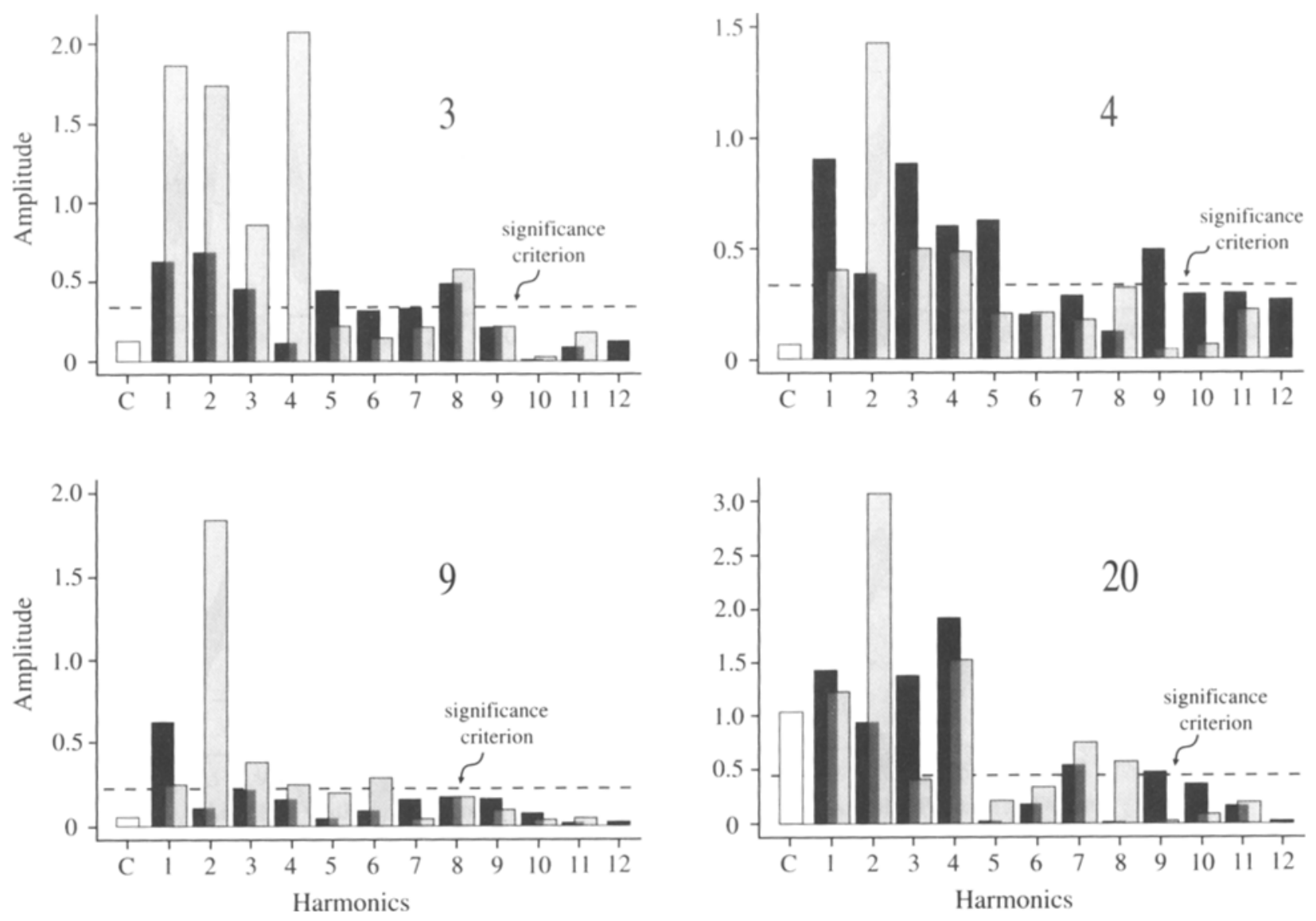

Figure 7. Harmonic analyses of the raw data yielded bar graphs that show a differential pattern of amplitudes for each subject. These provide spectral representation of the data from Figure 6, with cosine components shown in black, sine components in light gray, and the constant in white. Significant components are those for which the associated probabilities are $p<.15$, shown here with dashed lines.

quite similar. Whether or not a coefficient is included in the model is a function of its magnitude, its estimated variance, and its correlation with other coefficients. Generally, we found that each coefficient that was selected on the basis of the Mallows's Cp statistic had a Wald statistic (the ratio of amplitude to standard error) that was significant at $p<.15$. The "significance" lines shown in Figure 7 reflect the amplitudes that meet this probability level, and the coefficients that were above this line were included in the models described below. Note that the amplitudes that meet the criterion for significance vary from one subject to another, just as do the amplitudes themselves.

Figure 8 shows the inverse Fourier models for the 4 example subjects. To be specific, the coefficients selected by the $\mathrm{Cp}$ criterion were used to generate predictions of bias at each of the angular positions. We then estimated a $95 \%$ confidence interval for each prediction using the estimated covariance matrix of the coefficients. This incorporates the tendency for smaller variance at cardinal angles. Finally, because each model derives from sine and cosine components that have continuous distributions, we interpolated to intermediate angular positions, thus pro- viding smooth curves for the model boundaries. For convenience, we describe the major deviations of the model as delta oscillations.

It is clear that the profile of the delta oscillations is different for each of the example subjects (and, as demonstrated below, for each subject that was tested). In Figure 8 , we have added the mean that was observed at each angular position. Most of the means are contained by the model boundaries.

Figure 9 shows the models that were extracted for each of the 8 subjects from Experiment 1. The delta oscillations for Subjects 3 and 4 are presented again as part of the array. Any oscillation for which the confidence interval does not include zero is judged to be a significant effect. The subjects varied substantially in terms of the overall amplitude of oscillation, the direction (clockwise or counterclockwise) of the major oscillation within a given quadrant, and the degree to which the oscillation contained high-order harmonic components. We see no indication of a simple rule.

In Figure 10, we present the models for the subjects of Experiment 2, plotting the model for the first test day su- 

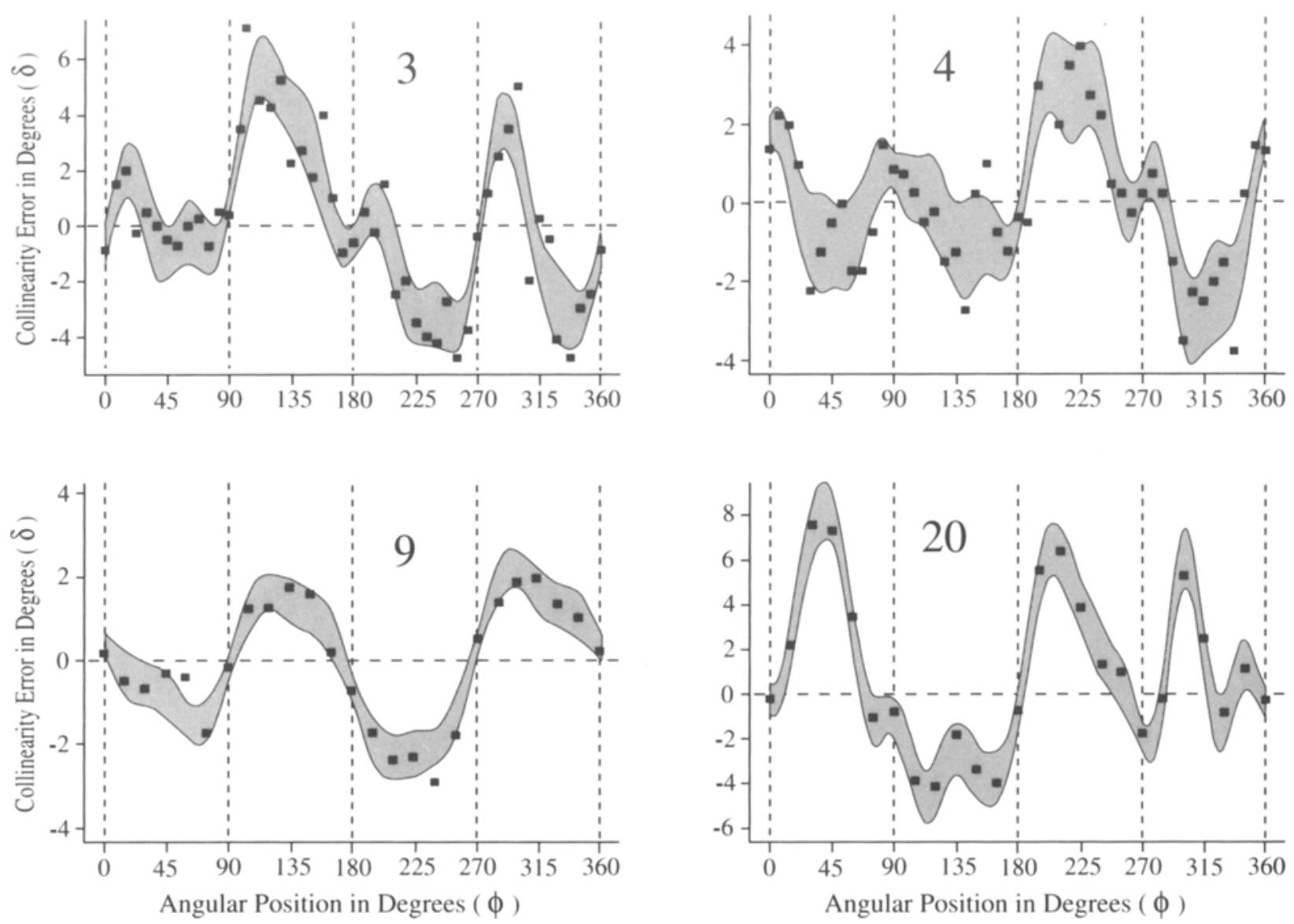

Figure 8. For each subject, the significant harmonic components are recombined to provide a model of the delta oscillation, and the subject's variance at each angular position provides the $95 \%$ confidence interval for the model. Mean judgment at each angular position is also plotted, and most of these values fall within this confidence interval.

perimposed on the model for Day 2. Here, we continue to see systematic and distinctive delta oscillations, but now we can see that the overall profile is very similar for each subject across the 2 test days. This suggests that the profile of the delta oscillation is not due to transitory test conditions but rather is a consistent and characteristic reflection of judgment tendencies for a given subject.

\section{Error Tendencies Differ Across Subjects But Are Stable Across Test Sessions}

We can formally address whether the error tendencies are distinctive for each subject by calculating a correlation matrix for the sample means from Experiment 2. To do this, we calculated a Pearson coefficient for Day 1 versus Day 2 for each subject, as well as the correlation of Day 1 for a given subject against Day 2 of each other subject and also the correlation of Day 2 of the subject against Day 1 for each other subject. (The other two combinations were calculated, and they yielded results comparable to those reported here.) These correlations are plotted in Figure 11. It can be seen that the correlation of Day 1 versus Day 2 for each subject is consistently at the .60 level or above and is higher than the correlation of that subject in relation to the data of other subjects.

We have estimated the probability that the self-self correlations would be consistently higher than any of the self-other correlations. Finding that it is the highest correlation among all the correlations for a given subject is analogous to drawing a red ball from an urn that otherwise contains black balls. Even though the draws are not independent, we estimate that the probability of sampling the red ball on 12 separate trials is no larger than $2.1 \times 10^{-9}$.

The substantial scatter of correlations across subjects, ranging generally from between \pm .70 , suggests that no particular profile of error tendency can be considered to be typical. Rather, the conclusion that seems to be warranted is that each subject has an idiosyncratic profile of judgment bias, which can be seen in the raw-data plot of delta against phi, or in the extracted model of the effect, as presented in Figures 9 and 10.

\section{Examining Significance of Coefficients}

In Figure 12, we have entered the Fourier coefficients for Experiment 1 and for the first day of Experiment 2, 
COLLINEARITY ERRORS

1449
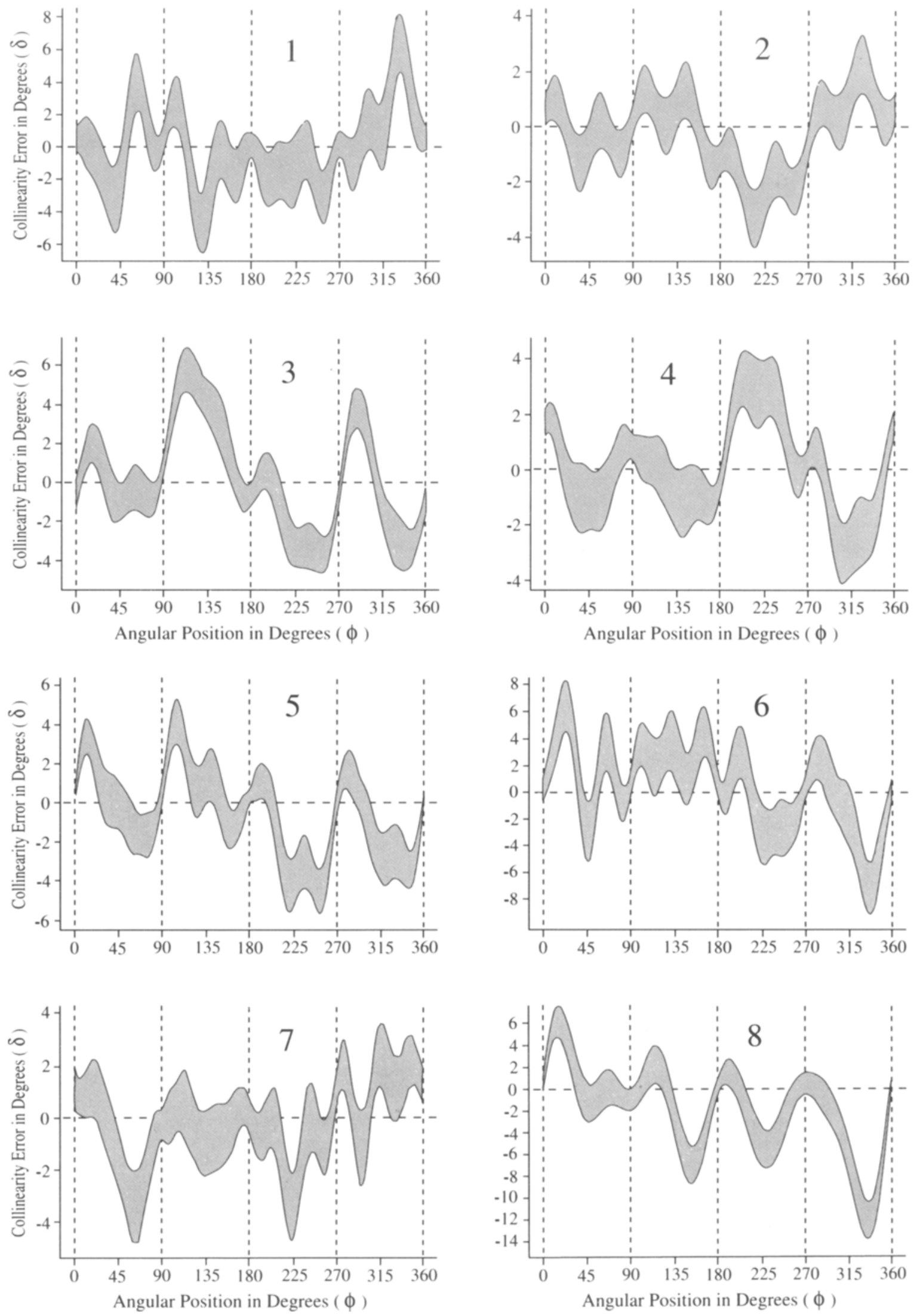

Figure 9. The model of delta oscillation is shown for each subject of Experiment 1. Note that each displays an idiosyncretic profile of error bias. 

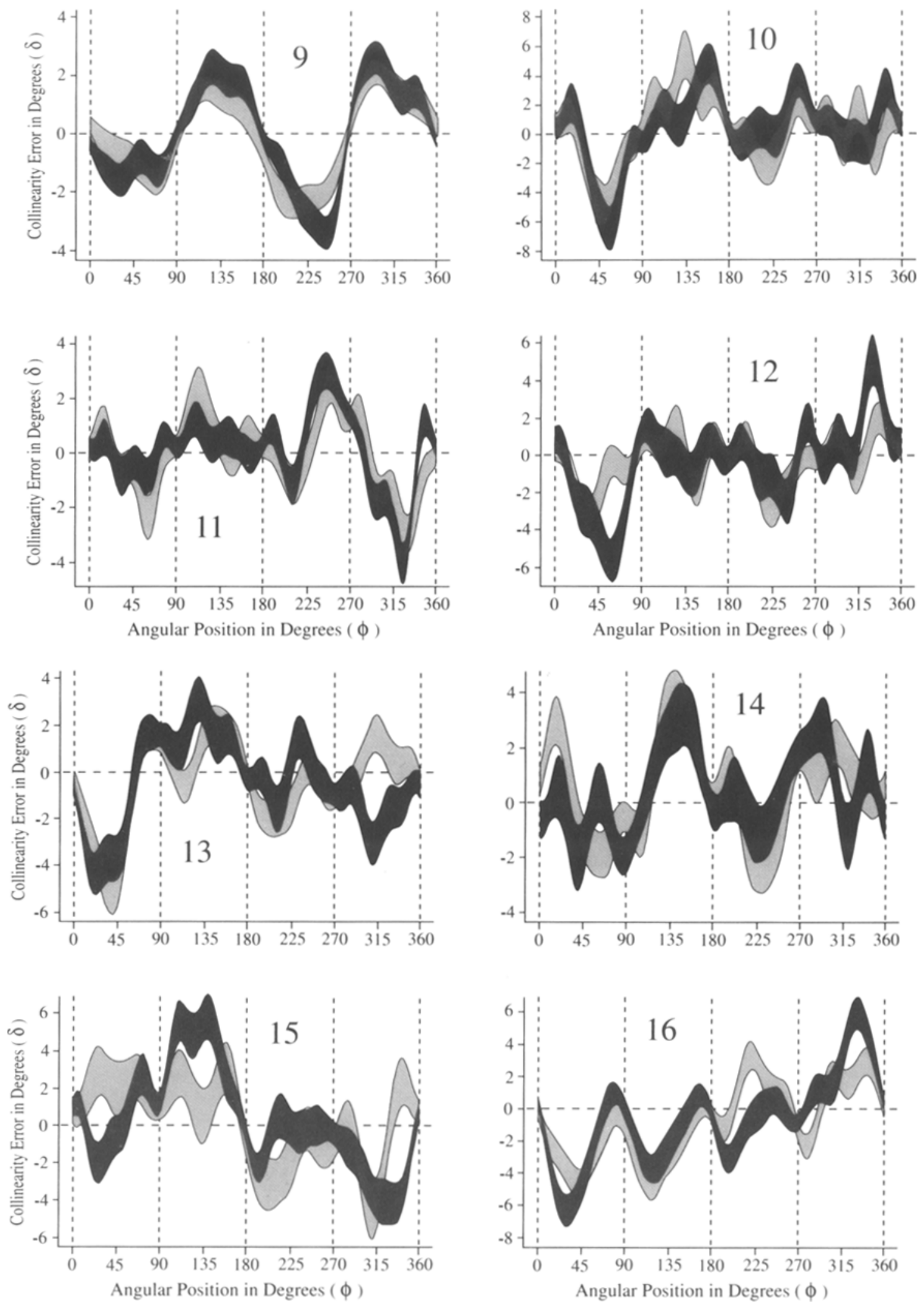

Figure 10. For the subjects of Experiment 2, the model for the first test day is shown as a gray band, and the model for the second test day is shown in black. The delta oscillations are different for each subject, but, for a given subject, the profile is similar from one test day to the next. (Additional panels for this figure are shown on the following page.) 

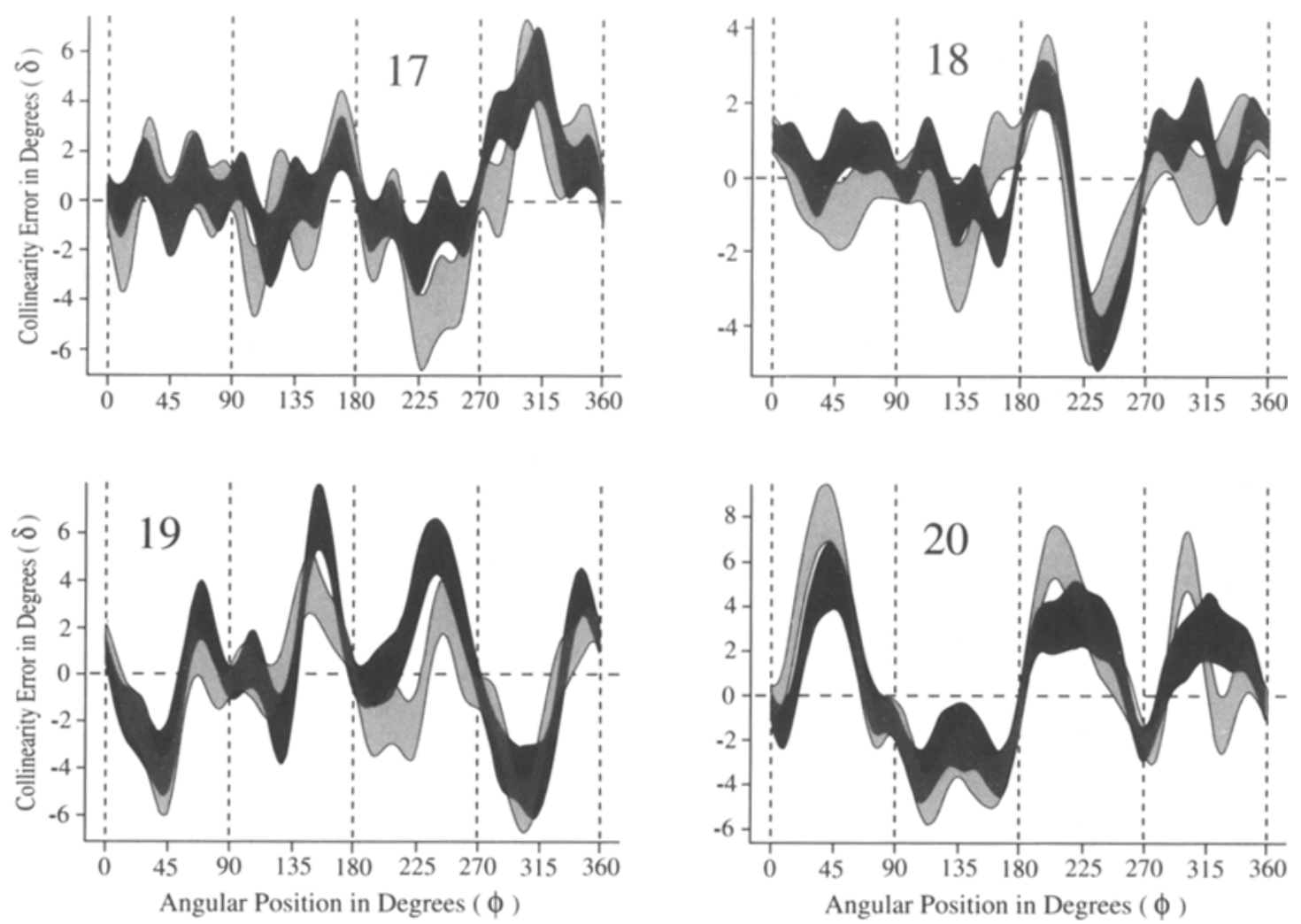

Figure 10 (continued).

these being the values that specified the models on the basis of our use of the $\mathrm{Cp}$ statistic. The values in the white cells were significant at $p<.15$. The values in the gray cells were significant at $p<.01$. The values shown in bold type (and in gray cells) were significant at $p<$ .0001 . Empty cells were nonsignificant.

Although our use of the Mallows statistic for model inclusion puts the threshold at $p<.15$, most of the coefficients shown in white in Figure 12 have associated probabilities that are considerably smaller. Taking an average of the probabilities associated with the white cells of Figure 12, we find that the mean probability is .06 .

We counted the number of significant coefficients for each harmonic component (across subjects) and show these counts in the left panel of Figure 13. In the right panel, we show the mean amplitudes (ignoring sign) for each harmonic component, these values being calculated using only the coefficients that met the minimal criterion for significance.

In Figure 12, subject-to-subject variation is manifested by size, sign, and distribution of the coefficient loadings. Across the 20 subjects, the average number of significant coefficients (out of 24 possible) was 10.6 , with a minimum of 5 (Subject 2) and a maximum of 15 (Subject 19). There was no harmonic component that had significant coefficients for every subject nor in which the sign was consistent. The $2 \mathrm{nd}, 3 \mathrm{rd}$, and 4 th harmonic sine compo- nents had the greatest number of loadings, with 16,17 , and 16 subjects (out of 20 ) having significant coefficients for these respective components. The 1 st harmonic cosine component was in this same range, in that 15 of the 20 subjects had significant coefficients. Indeed, if one considers the sine and cosine components together, one can see that most of the significant coefficients were in the 1st through the 4th harmonics, which will be elaborated on below. This is reflected in Figure 13, which shows that the number of significant coefficients drops sharply above the 4th harmonic (left panel), as does the mean amplitude of these coefficients (right panel).

There was no indication that the sign of the coefficients was consistent for any given harmonic or subject. We can note that 13 of the 16 at the $2 \mathrm{nd} / \mathrm{sin}$ component were negative. However, only 4 of 11 were negative for the $1 \mathrm{st} / \mathrm{sin}$ component, and only 5 of 16 were negative for the $4 \mathrm{th} / \mathrm{sin}$ component. If one considers the pattern of significant loadings across components for each subject, as well as their amplitudes and sign, one sees little indication of a common tendency. This is consistent with the broad spectrum of correlations shown in Figure 11.

\section{Should Harmonics}

\section{Above the 4th Be Discounted?}

It appears that the major source of judgment bias may be reflected in the 4 th harmonic and below. By far the 


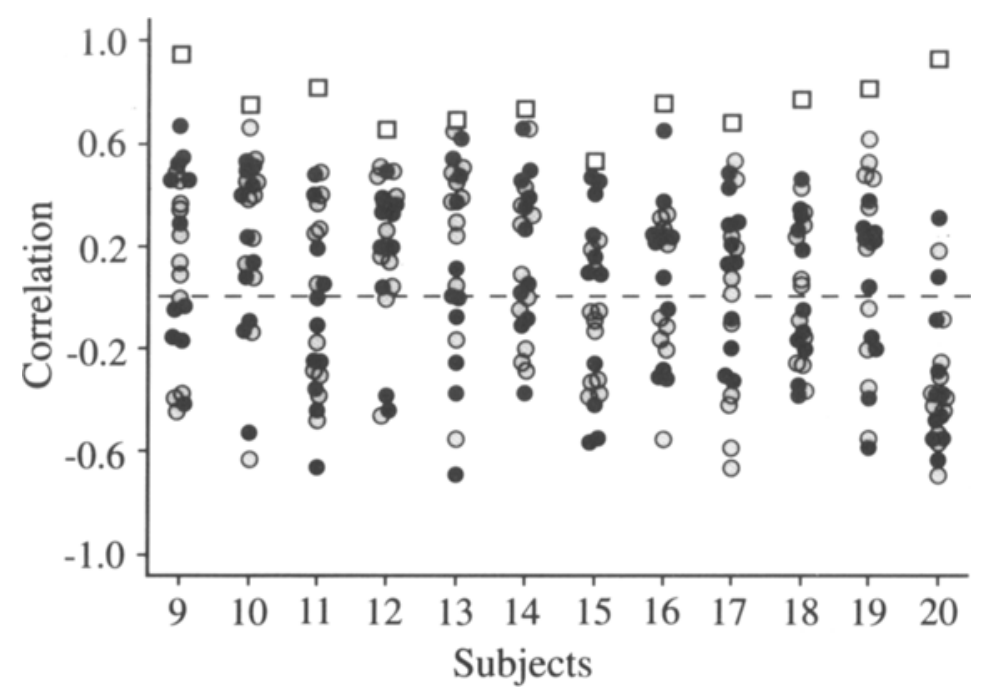

\begin{abstract}
Figure 11. Sample means at each phi from the 2 days of testing in Experiment 2 were correlated. The correlations for Day 1 with Day 2 for the same subject (open squares) were consistently higher than the correlations of that subject's data with the data of other subjects. (Day 1 of a subject with Day 2 of other subjects is shown in light gray, and Day 2 of a subject with Day 1 of other subjects is shown in black.)
\end{abstract}

greatest number of significant coefficients are found in the first 4 harmonics (and the constant). The count of coefficients that add or subtract at least $1^{\circ}$ (a tabled value of 1.00 ) to the delta error shows that only 6 of the 85 values are above the 4 th harmonic. Alternatively, one might tally those coefficients for which the calculated probability of error is $p<.0001$ (those with bold type and in the gray cells of Figure 12). Of the 64 that satisfy this stringent criterion, only 3 are above the 4 th harmonic.

Nonetheless, we are reluctant to claim that the higher coefficients have entered the table on the basis of chance. It should be remembered that all of the coefficients shown in Figure 13, including those above the 4th harmonic, are significant at a minimum of $p<.15$. Most are considerably higher than this value. If one considers only the significant coefficients at the 5th harmonic and above, the average of the associated probabilities is $p=.04$. Even if one wished to apply a $p<.05$ requirement before claiming significance, more than half of the coefficients at the higher harmonics would meet this criterion.

More specifically, across the 20 subjects, there are 30 coefficients at the 5 th harmonic and above that are significant at $p<.01$. These are scattered across the harmonics (e.g., 7 at the 5th; 9 at the 8 th). Though most of the coefficients make a small contribution to delta error, as can be seen in the tabled values, it is unlikely that they appeared on the basis of chance fluctuations of the data. For example, the $12 \mathrm{th} / \cos$ coefficients for Subjects 10 and 11 are only -.63 and -.35 , respectively, but they carry significance levels of $p s=.0011$ and .0006 , respectively.
At this early stage of inquiry, it does not seem wise to conclude that these are chance effects.

\section{Is the Coefficient Selection Too Lenient?}

Statistical studies of the Mallows's Cp statistic and other selection procedures suggest that they effectively discriminate signal from noise and thus specify a suitable model of treatment effects (see Hocking, 1976). In setting the criterion at $p<.15$, we are specifying the possibility that the value we observed was actually a chance departure from a mean of zero. Many researchers would judge this to be too lax a criterion. In many applications, the statistic is not considered significant unless $p<.05$ or, more stringently, $p<.01$.

In fact, the delta oscillations that are plotted in Figures 9 and 10 have profiles that are determined mostly by the high amplitude coefficients, many of these being significant at $p<.01$ and above. To illustrate this point, in Figure 14, we have replotted models for the subjects who served as examples in Figures 6-8. The light gray curves show the delta oscillations for models employing coefficients selected using Mallows's Cp statistic. Thus, each coefficient could have a probability of up to .15 of actually being zero. Each is superimposed on a black curve, which shows the delta oscillations obtained by using coefficients that were significant at $p<.01$.

By inspection, it is clear that the models are very similar. There is no indication that the use of a more stringent criterion for inclusion of coefficients changes the basic profile of each subject's delta oscillation, nor does it alter 


\begin{tabular}{|c|c|c|c|c|c|c|c|c|c|c|c|c|c|c|c|c|}
\hline & त: & & & & 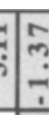 & ㅇ. & & & & & 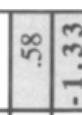 & & : & & & \\
\hline & a & & & ?. & $\stackrel{\infty}{\infty}$ & f. & \begin{tabular}{lll}
0 \\
\hdashline \\
\hdashline & 0
\end{tabular} & & & & & भ̂? & & $\stackrel{0}{0}$ & & | \\
\hline & $\infty$ & | & & $\stackrel{0}{\infty}=$ & 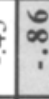 & 웅 & $\begin{array}{lll} & & \\
& \end{array}$ & & & הָ & (1) & f. & ণุ & & & \\
\hline & \pm & & & ț & $\overbrace{-\infty}^{\infty}$ & & & & & & & $\stackrel{\infty}{7}$ & & $\begin{array}{lll} & \\
\end{array}$ & & $\bar{\square}$ \\
\hline & $=1^{8}$ & & 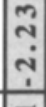 & t. & & \begin{tabular}{|l|}
0 \\
$\vdots$ \\
\hdashline \\
\end{tabular} & $\stackrel{7}{\stackrel{2}{*}}$ & $\bar{n}$ & 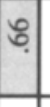 & & & : & $\overline{7}$ & & & \\
\hline & 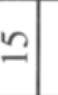 & & $\vec{a}$ & & $\stackrel{\infty}{n}$ & 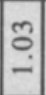 & 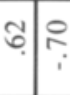 & & & $\stackrel{\infty}{?}$ & $\bar{\gamma}$ & : & & & & \\
\hline & \pm & & & 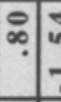 & & 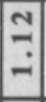 & & بִ & & \begin{tabular}{l|l}
$\circ$ & $\infty$ \\
$:$ &
\end{tabular} & & & & లి & & \\
\hline & $m=$ & 5 & & m: & & 잉 & & ? & के & & & & & & & \\
\hline & $\simeq$ & & & 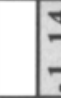 & & ? & 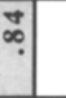 & & $\stackrel{\infty}{?}$ & ले & & ? & $\bar{n}$ & \begin{tabular}{c}
7 \\
\hdashline \\
\hdashline
\end{tabular} & & \\
\hline & $=$ & $\infty$ & & : & $\stackrel{5}{\hat{-}}$ & $\vec{\infty}$ & \begin{tabular}{l|l} 
& 7 \\
$\infty$ & 7
\end{tabular} & $\infty$ & ?ุ & ले & & & ?. & & ণิ) & $\begin{array}{l}m \\
i \\
i\end{array}$ \\
\hline$\stackrel{n}{n}$ & 의 & 7 & & & 2 & . & ఫ़ & & t. & 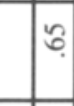 & \begin{tabular}{|l|}
\multirow{2}{*}{} \\
\end{tabular} & & & o. & & ?: \\
\hline 递 & $a$ & in & & $\because=$ & & m. & $\hat{\overbrace{}}$ & & & స̦ి & & & & & & \\
\hline मे & $\infty]^{\prime}$ & & ?. & 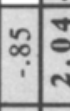 & & 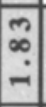 & \begin{tabular}{l|l} 
\\
\hdashline
\end{tabular} & & & $=\stackrel{-2}{-}=$ & $?$ & & & & & \\
\hline & r & el & 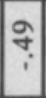 & 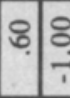 & ? & & ?. & $\approx$ & & $m$ & & & f. & ? & 年 & \\
\hline & 6 & 12 & ă & & $\stackrel{\circ}{:}$ & $\begin{array}{l}0 \\
\vdots \\
- \\
-\end{array}$ & $\stackrel{\hat{l}}{\hat{i}}$ & & $?$ & ?ִ. & \&. & $?$ & & ণ்ָ & 6 & \\
\hline & $n=$ & & $\begin{array}{l}0 \\
0 \\
-1 \\
-1\end{array}$ & 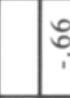 & & 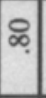 & $\infty$ & & & & & - & & & 7 & \\
\hline & $\nabla$ & ? & ণָे & 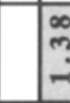 & $\bar{a}$ & जे & : & $\widetilde{\sigma}$ & & & & ले & רִ & & & \\
\hline & $\mathrm{m}$ & & $\begin{array}{l}2 \\
- \\
-\end{array}$ & & & $\ddot{0}$ & î & $\tilde{n}$ & & లె! & & กิ? & & & & \\
\hline & 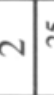 & & q. & $\stackrel{N}{-}$ & & & & & & & & 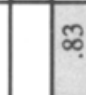 & & & & \\
\hline & & 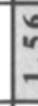 & & $\stackrel{\Delta}{\prime}$ & & กิ. & \begin{tabular}{l|l}
$\stackrel{\infty}{\circ}$ \\
\end{tabular} & & & ?ี & f. & @్ర & $\widehat{c}$ & & $\overline{5}$ & ?ִ \\
\hline & & & ["ळ] & ठै. & है & & $0]^{5}$ & 8 & के & 巳ั & ठ̊. & है & 引 & อั & D. & : \\
\hline & & & $\vec{\omega}$ & ปี & 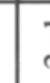 & ᄅ & 売 & & $\bar{r}$ & కే & 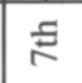 & $\stackrel{5}{\infty}$ & 5 & 홍 & $\stackrel{\Xi}{\Xi}$ & 䓌 \\
\hline
\end{tabular}



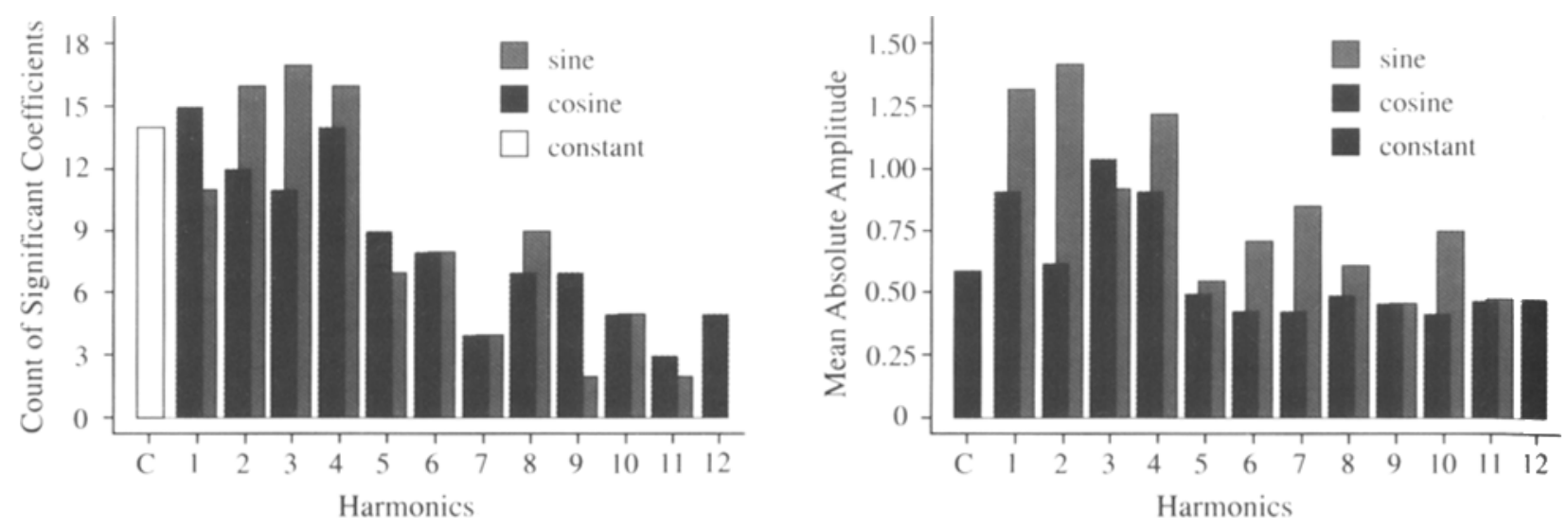

Figure 13. A count of the significant coefficients from Figure 12, for each harmonic component, is plotted in the left panel, and the mean amplitude (ignoring sign) is plotted on the right. The plots show that there are substantially more significant coefficients below the 5th harmonic, and they tend to have larger amplitudes.

the general conclusion that the profile is unique for each subject.

\section{DISCUSSION}

Our results show a clear cardinal axis differential with respect to error variability, in that the variability was minimal when the segment was aligned with a cardinal axis and maximal when the segment had an oblique tilt. This appears to be completely consistent with earlier work.

Sulzer and Zener (1953) measured orientation discrimination in 5 subjects by having them set a second segment parallel to the test segment. Variability of the orientation judgments was minimal when the test segment was horizontal, next best when it was vertical, and maximal when it was at an oblique orientation. Rochlin (1955) reported similar results.

Onley and Volkmann (1958) also found cardinal axis superiority for a task in which 12 subjects set a second segment to be perpendicular to the test segment. They plotted variance using polar coordinates, getting a clear four-leaf-clover pattern across three task conditions (4 subjects for each). We estimate that their plot would at least approximate a cycloid if it were rendered in Cartesian coordinates.

Bouma and Andriessen (1968) required a collinearity judgment, wherein the observer adjusted the position of a dot until it appeared to align with a test segment that was presented at various angular positions. They tested 3 subjects, at $15^{\circ}$ increments of angular position, in Quadrant 4 only. Their observed standard deviations plotted as cycloid across one period, and, on the basis of this result, they suggested a model that is similar to what we show in the lower right panel of Figure 3.

One reviewer has suggested that the cyclic variability across quadrants might be due to our use of rectangular test sheets, wherein the horizontal and vertical edges might serve to stabilize judgments at corresponding angular positions of the segment. We think this to be unlikely. A number of the studies have found an oblique effect using a round stimulus display field (e.g., Bouma \& Andriessen, 1968). Furthermore, subsequent experiments from this laboratory (Greene \& Frawley, 2000a, 2000b) have used a round display field with one end of the test segment (or one member of a dot pair) being anchored at the center of the display. The perceptual effects are similar to those reported here, in terms of both variability of response and idiosyncratic profiles of delta oscillation.

In discussing potential neural substrates for their data, Bouma and Andriessen (1968) invoked the concept that the stimulus segment is encoded by orientation selective cells in the cortex and suggested that there may be a greater number of cells whose receptive fields are aligned with a cardinal axis. Interestingly, they offered this hypothesis to explain their finding of intrinsic bias (discussed below), but it seems more appropriate with respect to the cycloid pattern of variance effects. A number of neurophysiologists have reported that there are more cells that prefer a cardinal orientation than that prefer an oblique orientation (De Valois, Yund, \& Hepler, 1982; Heggelund \& Albus, 1978; Leventhal \& Hirsch, 1980; Mansfield, 1975; Pettigrew, Nikara, \& Bishop, 1968). If the stability of perceptual encoding is a function of the number of cells that simultaneously register the stimulus, then a greater abundance of cells that have their receptive fields aligned with a cardinal axis could provide for less variability of response from one trial to the next.

Our results are also consistent with prior research, in showing that judgments are more accurate (in addition to being less variable) when the test segment is aligned with a cardinal axis. Prior studies were cited in the introduction, and we have nothing to add to what these investigators reported. Of greater interest was the finding of distinctive, idiosyncratic profiles of collinearity error as a function of angular position. Harmonic analysis of these delta oscillations yielded a unique pattern of coefficients 

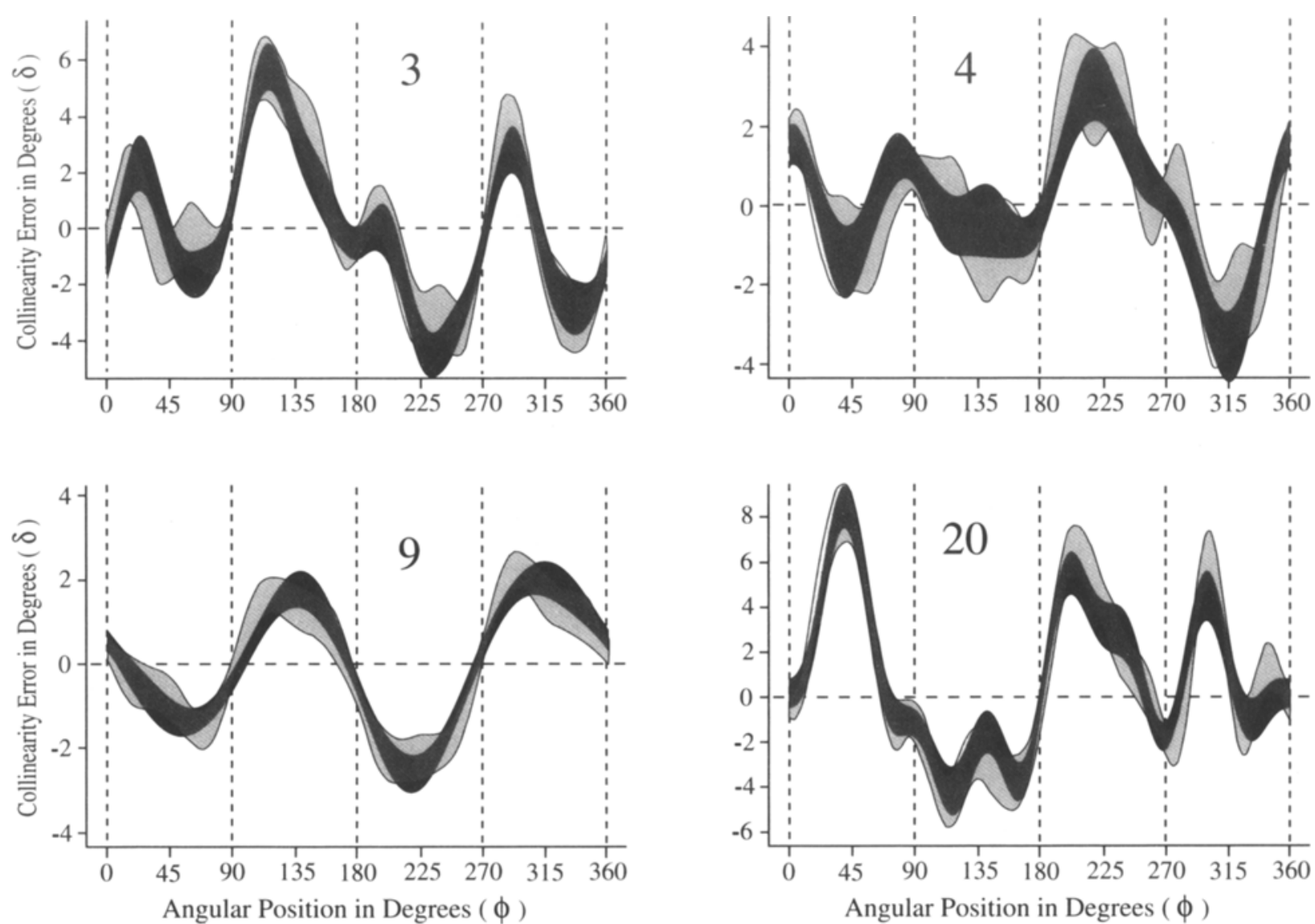

Figure 14. At the onset, we used these 4 subjects to illustrate the modeling method (Figures $6-8$ ). Here, we have remodeled their data to show the effect of using a more stringent significance criterion. As before, the light gray curves are derived from coefficients that meet $p<.15$. The black curves use coefficients that meet $p<.01$. The differences appear minimal.

for each subject, in terms of which harmonic components were significant, as well as the size and sign of those that were significant. Experiment 2 demonstrated that the delta oscillations were consistent from one test day to the next, in that self-correlations (same subject, Day 1 with Day 2) were consistently larger than cross-correlations (i.e., across the data of different subjects). Thus, each subject manifests a unique profile of delta bias as a function of angular position that is idiosyncratic to that individual.

Examining the literature in retrospect, we find a number of articles that hint at this outcome. In one of Jastrow's (1893) experiments, subjects adjusted a segment until it matched the orientation of a test segment that had been presented a few moments before. The tabled data show substantial variation of accuracy across test subjects as a function of orientation, which Jastrow notes with the following comment: "The errors vary with each angle and almost with each individual."

Leibowitz et al. (1955) evaluated the ability of subjects to judge the angular position of a stimulus dot that was briefly flashed, this being followed by an angular grid that provided a reference for judgments. They reported only positive error values, so presumably they were measuring absolute deviations. Their plot of error levels showed a complex pattern across angular positions, with greatest accuracy at cardinal positions and with the errors at oblique positions ranging up to $10^{\circ}$.

Onley and Volkmann (1958) asked subjects to adjust one segment to be perpendicular to another. Three sets of 4 subjects were tested, each judging a different configuration of the two segments (i.e., forming a $\mathrm{T}, \mathrm{L}$, or $\mathrm{X}$ ) and with the orientation of the stimulus configurations being varied. They reported that the profile of error bias (across orientations) was different for each subject for two of the stimulus configurations (the $\mathrm{T}$ and $\mathrm{L}$ ) and supported this claim with data plots from 5 of the 8 subjects. For the $\mathrm{X}$ configuration, they plot all 4 subjects and claim that the error profiles are similar. However, we see substantial differences in the curves that suggest idiosyncratic error tendencies.

Regan and Price (1986) used themselves as subjects to measure orientation difference thresholds for gratings. They varied the orientation of the grating in $7.5^{\circ}$ increments and found discrimination to be best for horizontal 
and vertical orientations, with weak submaxima at $45^{\circ}$. They noted, however, that the pattern of sensitivity across the angular spectrum was very complex and was different for the 2 observers.

The 8 subjects tested by Sittig and De Graaf (1994) were presented with three dots and were required to adjust the center dot until the three appeared to be aligned. They reported that the only consistent bias was a tendency to set the dot too low when the stimuli were presented in the first two quadrants. However, the plotted data (unfortunately superimposed into a jumble) appear to show substantial oscillation for a given subject, with the profile of the oscillation being different from one subject to the next. In follow-up experiments, using just a few subjects, they varied stimulus size, location of adjustable dot, and head tilt. Several figures plot a representative subject to illustrate the effect of each treatment. Each plot shows a complex profile of oscillation, being similar for the subject irrespective of the treatment being administered. It seems likely that each oscillation was reflecting intrinsic sources of bias for the subject, similar to what we have found in the present study.

Bouma and Andriessen (1968) claimed support for a cardinal assimilation hypothesis on the basis of two experiments. In the first, measures were taken from 3 subjects across seven angular positions in one quadrant. They observed judgments to be fairly accurate when the segments were horizontal, vertical, or at $45^{\circ}$. At the intermediate positions, the segments were judged as lying closer to the nearest cardinal axis than was true. These effects were modeled as $\beta=-B \sin 4 \alpha$. A second study tested 2 subjects at angular positions for which the model predicted maximal effect - specifically, $22^{\circ}, 68^{\circ}, 112^{\circ}, 158^{\circ}$, $202^{\circ}, 248^{\circ}, 292^{\circ}$, and $338^{\circ}$. Error levels were alternately high and low, more or less matching the peaks of their model.

One might object to conclusions that are based on so few subjects, and especially with the suggestion that a model is properly tested with a sampling that barely meets the Nyquist limit. Aside from that, there was considerable scatter in their data, so it is possible or even likely that their model did not specify all sources of error. Five of our subjects had significant coefficients on the 4 th $/ \mathrm{sin}$ component, in the direction specified by the Bouma and Andriessen (1968) model. However, for 11 other subjects the 4th harmonic bias was in the opposite direction (i.e., positive). Furthermore, on average, the size of effect was larger for the positive coefficients. More to the point, all subjects had a large number of significant coefficients at harmonics not specified by their model.

A major issue is the question of how to interpret the bias that is reflected in a significant coefficient. The role of the constant could be straightforward. Here, we are talking about a uniform rotation of judgments, counterclockwise if positive and clockwise if negative. Thus, a +.50 constant coefficient means that judgments tend to lie $0.5^{\circ}$ counterclockwise to true collinearity across the full spectrum of angular positions.

For the first harmonic or two, one must consider rectilinear response tendencies. For example, if there is a tendency to select a target toward the left side of the sheet, this will be registered as counterclockwise error in the upper half and clockwise error in the lower half. The result would be a positive 1 st harmonic sine component. Similarly, a tendency to reach too low on the sheet generates a negative 1 st harmonic cosine component.

One can formulate more complex reaching tendencies and/or hypothesize that there is assimilation to (or from) one or both cardinal axes. With such influences, one might expect to see significant coefficients at the 2 nd harmonic and the 4th harmonic. However, as the harmonic number rises, it becomes increasingly difficult to view the process in rectilinear terms. Except for the 4th harmonic and perhaps the 8 th, we cannot envision a simple rectilinear model that would generate coefficients at any of the higher harmonics. Even if one wanted to consider only effects for the 1st, 2nd, and 4th harmonics, it is difficult to see what source of lateralized response would produce combined loadings on all three. In the absence of evidence to the contrary, therefore, we are inclined to accept the coefficients as reflecting angular position error per se.

Furthermore, the lack of consistent loadings for any harmonic component argues against a simple theory of perceptual bias. Across subjects, there was no indication of consistent assimilation or contrast with respect to cardinal axes. The studies that reported assimilation or contrast may have used stimulus configurations or testing procedures that biased the results in that direction. Where results were based on just a few subjects, these individuals might have been alike, by chance, with respect to a particular harmonic component. Alternatively, where groups of subjects were considered, the data summary may have averaged out all but a small common residual, which then was claimed as the basic effect. Whatever the case, our results do not suggest any cardinal axis assimilation or contrast as part of the basic perceptual mechanism.

Previous work from this laboratory supports the view that the collinearity error is properly measured as a deviation of angular position and also speaks to the issue of whether the effects are from the perceptual or from the motor system. The most direct evidence is provided by Greene $(1993,1994)$, in which the stimulus configuration and test conditions were essentially identical to the present methods, except that a second "induction segment" was added at the tip of the segment being judged. The induction segment produced a large bias of collinearity judgments that was a simple function of the relative angle between the two segments. For a specific induction angle, the collinearity error was found to be a constant angle irrespective of the distance at which the subject responded. In other words, the induction segment produced an angular position error rather than a rectilinear error. 
Additionally, Greene (1993) found that the size of the induction error was the same whether measured by the operant method of dot placement (used here) or by having the subject decide whether or not a prepositioned dot was collinear. The latter procedure is essentially a "method of constant stimuli," wherein the decision relates to the perceptual state that is induced by the stimulus configuration and has no spatial-motor demand. A similar evaluation of operant versus perceptual response modes has been done for judgments of linear extent (Nelson \& Greene, 1998), and here also there was no indication of motor bias. We think it likely, therefore, that the operant procedure of selecting and marking a collinear position accurately reflects intrinsic sources of judgment bias and that the delta oscillations provide a model of the biased perceptual state.

The basis for individual differences in delta oscillation is not clear. Optical defects seem unlikely for at least two reasons. First, it is doubtful that 20 young subjects would all have clinical defects that had gone unrecognized. Second, there is no reason to expect optical defects to be more severe for oblique axes and less severe for cardinal axes.

It is possible that each subject adopts a slightly different fixation point when examining the stimulus configuration and that the location of the fixation is a factor in the size and sign of error across the spectrum of angular positions. This seems at odds with the observation that subjects were significantly more accurate (and had lower variability) when the segment was aligned with a cardinal axis. At the very least, one would need to specify that subject-by-subject differences are specific to fixation of oblique (i.e., noncardinal) angular positions.

Another possibility is that the errors reflect defects in the neural systems that encode the stimulus segment and/or the location of potential collinear target sites. It is likely that the registration of the stimulus, and perhaps the encoding of its position, is mediated by neural receptive fields in the retina and at higher visual centers. The analysis of stimulus position may depend on the correct positioning of receptive fields, and the present idiosyncratic error tendencies may reflect flaws in anatomical development. The present data shed no light on whether this might be a valid hypothesis, and, therefore, we resist the temptation to speculate further.

In closing, we would like to draw a bright line between the methods for modeling errors and the speculation about possible mechanisms. Harmonic analysis, which was the basis for modeling, is essentially "theory neutral." It is a useful and efficient method for capturing patterns of deviation in the data, but it is unlikely that the harmonic components themselves correspond to physiological substrates. A given harmonic component, by its nature, specifies the extent to which a periodic oscillation is present in the data. If the process being modeled has this same period, then the amplitude of the component will reflect the contribution of this process. However, if the source of error is more local, or if it is the sum of local defects, then one should not attribute any functional significance to the harmonic component.
The value of the present research is in rejecting older claims of assimilation (or contrast) with respect to cardinal axes, in establishing that earlier single-component models of bias are erroneous, and in showing that there are substantial individual differences in the profiles of error with judgment of angular position/collinearity. If we are to make progress in understanding the sources of error and the mechanism behind the judgments, it will be necessary to use methods that can accommodate these individual differences. Notwithstanding the caveat about functional attribution discussed above, harmonic analysis appears to offer some potential benefits in dealing with this difficult issue.

\section{REFERENCES}

ANDREwS, D. P. (1967). Perception of contour orientation in the central fovea. Part 1: Short lines. Vision Research, 7, 975-997.

APPELLE, S. (1972). Perception and discrimination as a function of stimulus orientation: The "oblique effect" in man and animals. Psychological Bulletin, 78, 266-278.

Bouma, H., \& Andriessen, J. J. (1968). Perceived orientation of isolated line segments. Vision Research, 8, 493-507.

Craig, E. A., \& Lichtenstein, M. (1953). Visibility-invisibility cycles as a function of stimulus-orientation. American Journal of Psychology, 66, 554-563.

De Valois, R., Yund, E., \& HePler, N. (1982). The orientation and direction selectivity of cells in Macaque visual cortex. Vision Research, 22, 531-544

EMSLEY, H. H. (1925). Irregular astigmatism of the eye: Effect of correcting lenses. Transactions of the Optical Society, 27, 28-41.

Green, R. T., \& Hoyle, E. M. (1964). The influence of spatial orientation of the Poggendorff illusion. Acta Psychologica, 22, 348-366.

GreENE, E. (1993). The influence of response factors on the magnitude of angular induction. Perceptual \& Motor Skills, 76, 323-335.

GreEnE, E. (1994). Collinearity judgment as a function of induction angle. Perceptual \& Motor Skills, 78, 655-674.

GreEne, E., \& FraWley, W. (2000a). Evaluating models of collinearity judgment for reliability and scale. Manuscript submitted for publication.

GREENE, E., \& FraWLEY, W. (2000b). Idiosyncratic profiles of collinearity error using segments and dot pairs. Manuscript submitted for publication.

Heggelund, P., \& Albus, K. (1978). Response variability and orientation discrimination of single cells of cat. Experimental Brain Research, 32, 197-211.

HockING, R. R. (1976). The analysis and selection of variables in linear regression. Biometrics, 32, 1-50.

Hotopf, W. H. N., Ollerearnshaw, C., \& Brown, S. (1974). The regression to right angles tendency and the Poggendorff illusion. III. British Journal of Psychology, 65, 213-231.

JASTROW, J. (1893). On the judgment of angles and positions of lines. American Journal of Psychology, 5, 214-248.

JuDD, C. H. ( 1899). A study of geometrical illusions. Psychological Review, 6, 241-261

Leibowitz, H. W. (1953). Some observations and theory on the variation of visual acuity with orientation of the test object. Journal of the Optical Society of America, 43, 902-905.

Leibowitz, H. W., Meyers, N. A., \& Grant, D. A. (1955). Angular localization of a single stimulus as a function of luminance and duration of exposure. Journal of the Optical Society of America, 45, 76-78.

Leventhal, A., \& Hirsch, H. (1980). Receptive field properties of different classes of neurons in visual cortex of normal and dark reared cats. Journal of Neurophysiology, 43, 1111-1132.

MANSFIELD, R. (1975). Neural basis of orientation perception in primate vision. Science, 167, 1133-1135.

Nelson, B., \& GreENE, E. (1998). Similar Müller-Lyer effects from operant and comparison response modes. Perceptual \& Motor Skills, 86, 499-311. 
Onley, J. W., \& Volkmann, J. (1958). The visual perception of perpendicularity. American Journal of Psychology, 71, 504-516.

Orban, G. A., Vandenbussche, E., \& Vogels, R. (1984). Human orientation discrimination tested with long stimuli. Vision Research, 24, $121-128$.

Pettigrew, J., Nikara, T., \& Bishop, R. (1968). Responses to moving slits in cat striate cortex. Experimental Brain Research, 6, 373-390.

Regan, D., \& Price, P. (1986). Periodicity in orientation discrimination and the unconfounding of visual information. Vision Research, 26, 1299-1302.

Rochlin, A. M. (1955). The effect of tilt on the visual perception of parallelness. American Journal of Psychology, 68, 223-236.

SitTig, A. C., \& DE GraAF, J. B. (1994). Orientation dependent misalignments in a visual adjustment task. Vision Research, 34, 2195 2203

Sмiтн, S. L. (1962). Angular estimation. Journal of Applied Psychology, 46, 240-246.

Sulzer, R., \& ZENER, K. (1953). A quantitative analysis of relations between stimulus determinants and sensitivity of the visual perception of parallelness. American Psychologist, 8, 444.

VERNON, M. D. (1934). The perception of inclined lines. British Journal of Psychology, 25, 186-196.

Weintraub, D. J., \& Krantz, D. H. (1971). The Poggendorff illusion: Amputations, rotations, and other perturbations. Perception \& Psychophysics, 10, 257-264.

Weintraub, D. J., \& Virsu, V. (1971). The misperception of angles: Estimating the vertex of converging line segments. Perception \& Psychophysics, 9, 5-8.

WUNDT, W. (1893). Grundzüge der physiologischen Psychologie [Fundamentals of physiological psychology]. Leipzig: Engelmann.

\section{NOTES}

1. Operational definitions and terms of art are shown in italic at the point where they are most clearly specified.
2. With respect to these articles, the term angular position is equivalent to the angle parameter of a polar coordinate system. But for discussing relevant literature, it may be used in a generic sense, to include stimulus orientation

3. The meanings of the terms contrast and assimilation tend to vary with the phenomenon under investigation and, to some extent, with the theorist. With respect to geometric illusions, the terms are often used to imply a force of action that changes the position of stimulus elements: assimilation meaning that there is an attraction, and contrast meaning repulsion. Here, they are simply descriptive, to indicate that judgments have been shifted in a certain direction.

4. In prior studies of angular induction from this laboratory, we identified the orientation of the test segment as omega. With further reflection it seems most appropriate to restrict this term to task conditions in which orientation is judged (i.e., where the full range of rotation is $180^{\circ}$ ). In the present experiments, the stimulus varied across a $360^{\circ}$ range, so we adopt the convention of identifying these angular positions as phi.

5. An examination of absolute error, wherein sign is ignored, is required because subsequent analysis demonstrates that subjects have large nonrandom errors that can be either positive or negative. Calculation of a simple mean across all errors would allow these significant departures to cancel one another.

6. The bar graphs display absolute rather than signed amplitudes, for this allows the significance criterion to be shown as a single line. Note that this line is an approximation, since the significance of a given component varies somewhat as a function of its magnitude, standard error, and correlation with other components. For purposes of modeling, the sign of the coefficient was included in the combining of significant components.

(Manuscript received December 2, 1998; revision accepted for publication November 29, 1999.) 